\title{
UNA ESPECIE NUEVA DE ESCOBEDIA (OROBANCHACEAE), DEL ESTADO DE MICHOACÁN, MÉXICO
}

\author{
Eleazar Carranza González ${ }^{1}$ y Consuelo Medina García ${ }^{2}$ \\ ${ }^{1}$ Instituto de Ecología, A.C., Centro Regional del Bajío, Apdo. postal 386, 61600 \\ Pátzcuaro, Michoacán, México. \\ ${ }^{2}$ Universidad Nacional Autónoma de México, Centro de Investigaciones en \\ Geografía Ambiental. Morelia, Michoacán, México.
}

\section{RESUMEN}

Se describe como nueva a Escobedia michoacana (Orobanchaceae), encontrada en Michoacán, México. También se presenta una ilustración de la misma, una pequeña discusión de las posibles relaciones que guarda con otras especies del género y una clave para determinar los componentes de Escobedia que se reconocen para México. Se trata de una planta aparentemente escasa y restringida a la porción noroccidental del estado, aunque probablemente se encuentre también en Jalisco. Difiere de las demás especies mexicanas del género, por presentar cinco (no diez) nervaduras principales que corren a manera de costillas a lo largo del cáliz.

Palabras clave: Escobedia, México, Michoacán, Orobanchaceae.

\begin{abstract}
Escobedia michoacana (Orobanchaceae) is described as a new species from the state of Michoacán, Mexico. An illustration is also presented, together with a discussion of its affinities to other species and a key to distinguish the Mexican members of the genus. It is a rare taxon restricted to the northwestern part of the state, although it possibly occurs in adjacent Jalisco. It differs from the other Mexican species of the genus by possessing 5 (vs. 10) rib-like veins extending the length of the calyx.
\end{abstract}

Key words: Escobedia, Mexico, Michoacan, Orobanchaceae. 
Escobedia Ruiz \& Pavón es un género americano, tratado con anterioridad en la familia Scrophulariaceae y actualmente, con base en estudios moleculares (Olmstead et al. 2001), se ubica dentro de Orobanchaceae. La distribución conocida de estas plantas se extiende desde el norte de México hasta Argentina. No se han llevado a cabo trabajos detallados sobre este grupo en las últimas décadas y la revisión más reciente data de hace más de 75 años (Pennell, 1931), en la cual se reconocen 15 especies. Sin embargo, como sinónimos de E. grandiflora (L. f.) Kuntze, se menciona en el estudio de la familia Scrophulariaceae para Ecuador (Holmgren y Molau, 1984) a E. foliolosa Pennell, mientras que Ulloa (2006) hace lo mismo con E. scabrifolia Ruiz \& Pavón, en un tratado sobre el uso de plantas en los Andes. A pesar de que se entiende como un género sin mayores problemas taxonómicos, la proposición de las sinonimias anteriores y la acumulación de colecciones actuales más completas, que con seguridad albergan una cantidad considerable de ejemplares, sugieren la necesidad de preparar una revisión moderna del mismo.

Las especies citadas para México además de E. michoacana, son: E. crassipes Pennell, E. guatemalensis Loes., E. laevis Cham. \& Schltdl., E. longiflora Pennell y E. peduncularis Pennell. A reserva de aclarar la situación taxonómica de las plantas de Centro y Sudamérica, el número total hasta ahora aceptado de los miembros de Escobedia oscila entre 8 y 10.

Durante la preparación de la familia Orobanchaceae para la Flora del Bajío y de Regiones Adyacentes, se descubrió una novedad de este género, de la cual se encontraron unos cuantos ejemplares procedentes de la porción noroccidental del estado de Michoacán, México.

Escobedia michoacana E. Carranza \& C. Medina sp. nov. Fig. 1

Caulis herbaceus, simplex, $30-80 \mathrm{~cm}$ altus; folia lanceolata, (2)3-6(7) $\mathrm{cm}$ longa, 1-2.4 cm lata, dentata; pedunculi 5-10 cm longi, bracteae $1.3-2.8 \mathrm{~cm}$ longae, infra calycem 1.5-3.5(4.2) cm positae; calyx 5-costatus, 4.5-6.5 cm longus, glaber vel in costis strigosus; corolla $6.8-9.8 \mathrm{~cm}$ longa, externe glanduloso-puberula.

Planta herbácea, de 30 a $80 \mathrm{~cm}$ de alto; tallo blanco-piloso, los pelos de alrededor de $2 \mathrm{~mm}$ de largo; hojas opuestas, estípulas caedizas, láminas sésiles, lanceoladas, de (2.5)3 a 6(7) cm de largo, de 1 a $2.4 \mathrm{~cm}$ de ancho, ápice agudo a acuminado, base redondeada a subcordada, margen dentado, dientes agudos, 4 a 8(10) a cada lado, a veces hasta de $2 \mathrm{~mm}$ de largo, una vena secundaria basal a cada lado de la central, a veces una más que se desprende de cada una de las secundarias, el resto 

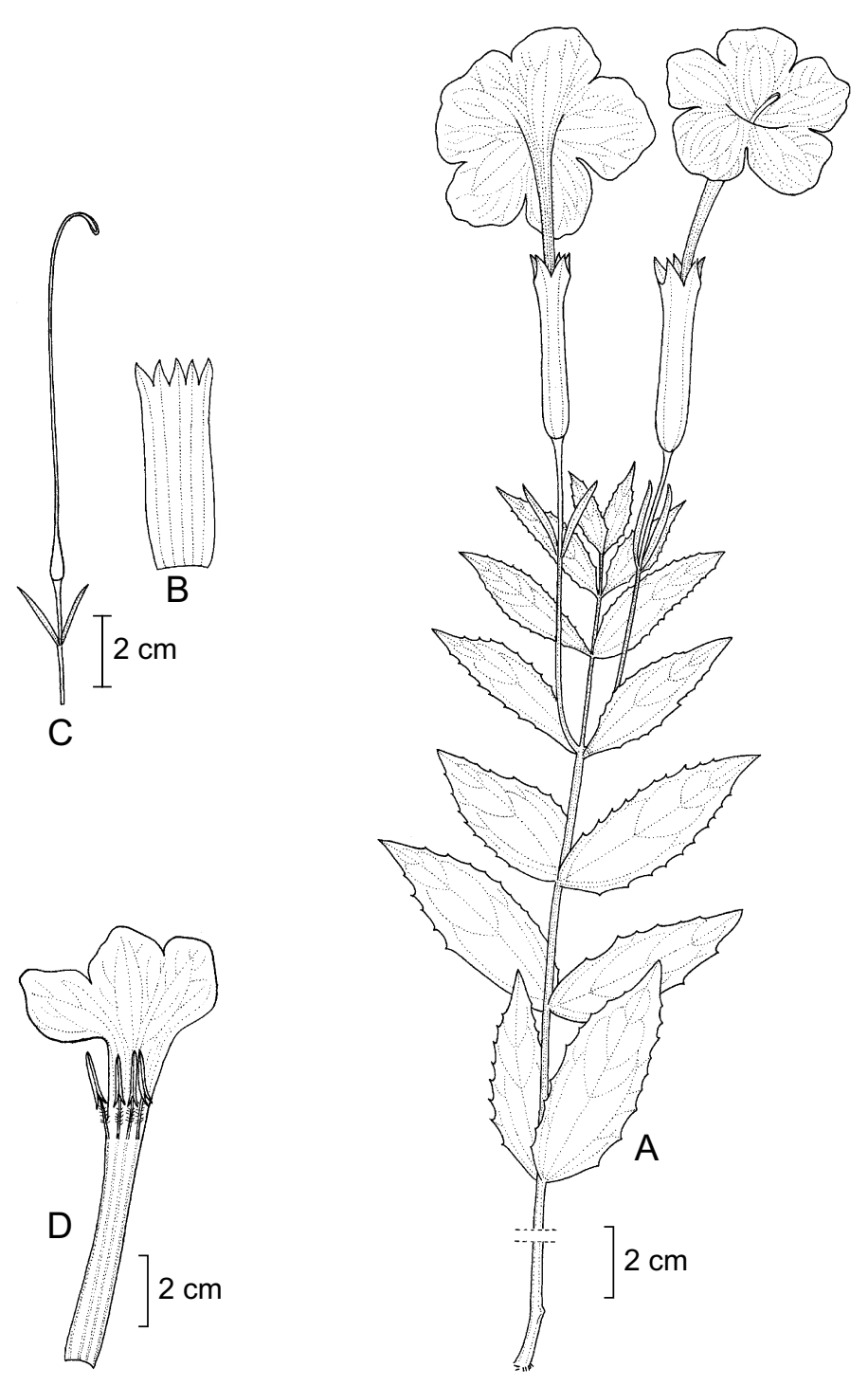

Fig. 1. Escobedia michoacana. A. rama; B. cáliz abierto mostrando las costillas; C. flor desprovista de los sépalos y corola, mostrando el estilo; D. corola abierta, mostrando la inserción de los estambres. 
de la nerviación reticulado, papiráceas a más o menos coriáceas, estrigosas, sobre todo en el haz; flores axilares, solitarias hacia el ápice de las ramas, pedúnculo de 5 a $10 \mathrm{~cm}$ de largo, glabro, brácteas 2, opuestas, situadas de 1.5 a $3.5(4.2) \mathrm{cm}$ abajo de la base del cáliz, lineares, a veces algo ensanchadas en la parte media, de $1.3 \mathrm{a}$ $2.8 \mathrm{~cm}$ de largo, de $1 \mathrm{a} 3 \mathrm{~mm}$ de ancho, glabras con el margen apenas hirsuto, sobre todo hacia el ápice, agudas; cáliz tubular, 5-dentado, 5-costillado (nervado), glabro o a veces las costillas esparcidamente estrigosas, de 4.5 a $6.5 \mathrm{~cm}$ de largo, dientes triangulares, de 7 a $10 \mathrm{~mm}$ de largo, agudos; corola hipocraterimorfa, de 6.8 a 9.8 $\mathrm{cm}$ de largo, blanca, tubo más o menos recto a curvado, de 2 a 4(5) $\mathrm{mm}$ de diámetro, parte interna superior del tubo $( \pm 2 \mathrm{~cm})$ y garganta pilosos, pubérulo-glandulosa en el exterior, limbo 5-lobulado, de 4.5 a $6.5 \mathrm{~cm}$ de diámetro; estambres 4, inclusos, filamentos adheridos al tubo de la corola, la porción libre pubescente y de 6 a 10 $\mathrm{mm}$ de largo, anteras de 1.5 a $1.6 \mathrm{~cm}$ de largo, lineares, la base sagitada, largamente apendiculada; estilo por lo general exserto, sobrepasando de 1.5 a $2.5 \mathrm{~cm}$ a los estambres, estigma a manera de una línea a cada lado de la porción apical del estilo, ésta un poco engrosada; cápsula ovoidea a elipsoidea, de alrededor de $3 \mathrm{~cm}$ de largo y $1 \mathrm{~cm}$ de diámetro, glabra; semillas no vistas.

Tipo: México, Michoacán: $2 \mathrm{~km}$ al SW de El Zapote, sobre la carretera a Villa Jiménez, municipio de Villa Jiménez, 17 Ago 2008, 2100 m s.n.m., pastizal, J. Rzedowski 54282 (holotipo: IEB; isotipos ENCB, MEXU, XAL).

Paratipos: México, Michoacán: El Llano del Chupadero, municipio de Churintzio o Zináparo, 2 Ago 1984, 1980 m s.n.m., J. N. Labat 1060 (ENCB); camino al cerro de La Cruz, municipio de Tlazazalca, 5 Jul 1990, matorral subtropical, E. Pérez y E. García 1351 (IEB); Presa La Yerbabuena, municipio de Tlazazalca, 8 Ago 1990, 1950 m s.n.m., E. Pérez y E. García 1590 (EBUM, IEB, MEXU, XAL); 5 km al E de Villa Jiménez, sobre el camino a Copándaro, municipio de Villa Jiménez, 3 Sept 1989, 2000 m s.n.m., J. Rzedowski 48878 (IEB); alrededores de La Soledad, municipio de Villa Jiménez, 17 Ago 2008, terrenos encharcados a la orilla de la carretera, J. Rzedowski 54280 (IEB); $6 \mathrm{~km}$ WSW of Jacona (on road to Los Reyes), municipio de Jacona, 29 Jul 1960, 2000 m s.n.m., H. H. Iltis et al. 525 (ENCB); aprox. 0.5 km al sur de San Francisco, carretera Jaripo - Cotija, municipio de Cotija, 9 Ago 1991, 1700 m s.n.m., I. García R. 3314 (CIMI, IEB); desviación a Los Tábanos, aprox. 1 km de la carretera Jiquilpan - Colima, aprox. 3-4 km de El Fresno, municipio de Jiquilpan, 25 Ago 2005, 1800 m s.n.m., I. García 7179 (CIMI, IEB). 
Escobedia michoacana se distingue fácilmente de los demás representantes mexicanos del género por las cinco nervaduras a manera de costillas que tiene en el cáliz, a diferencia del resto que tienen 10. Además, cada una de estas venas en la primera especie terminan en la punta de un diente del tubo calicino, mientras que en los otros taxa acaban alternadamente en senos y ápices de los lóbulos.

Al igual que el nuevo taxon, las plantas de Centro y Sudamérica (excepto E. guatemalensis), poseen el mismo número de cinco nervaduras en el cáliz; pero E. michoacana es distinta en el tamaño del pedúnculo y de las brácteas. La planta mexicana tiene el pedúnculo de 5 a $10 \mathrm{~cm}$ de largo, mientras que en las otras especies éste mide entre 0.5 y $6 \mathrm{~cm}$ de longitud. Sólamente en E. grandiflora (L. f.) Kuntze alcanza hasta $6 \mathrm{~cm}$, pero, el cáliz de ese taxon sudamericano es menor de 4 $\mathrm{cm}$ a diferencia del mexicano que lo tiene de más de $4.5 \mathrm{~cm}$ de largo.

Por otra parte las brácteas de E. michoacana son de mayor tamaño (13 a 28 $\mathrm{mm}$ de largo) que la mayoría de las de las plantas centro y sudamericanas. En estas últimas se han registrado medidas menores de $13 \mathrm{~mm}$ de largo, a excepción de $E$. foliolosa Pennell (14 a $20 \mathrm{~mm}$ ) y de E. grandiflora (L. f.) Kuntze (hasta de $30 \mathrm{~mm}$ ); sin embargo, ambas difieren de la especie de México en el tubo calicino más corto y en la mayor proximidad de las brácteas a la base del cáliz.

Sin lugar a dudas el carácter que relaciona a las plantas sudamericanas, así como las de Centroamérica con el nuevo taxon, es la posesión de cinco nervaduras (costillas) paralelas a lo largo del cáliz. Pero si se consideran otros rasgos morfológicos de este grupo, no resulta claro cuál o cuáles de los taxa del sur tienen mayor cercanía con la especie mexicana.

Las plantas de esta especie se han encontrado habitando cuerpos de agua, o por lo menos lugares con bastante humedad del suelo, generalmente en zonas con vegetación secundaria derivada de bosque tropical caducifolio y matorral subtropical. Por lo común se han registrado asociadas Proboscidea louisianica y representantes de los géneros Sagittaria, Scirpus, Cyperus y Zornia. La altitud donde se ubican oscila entre 1700 y $2000 \mathrm{~m}$.

En cuanto al sustrato edafológico (Anónimo, 1981, 1985), las poblaciones de E. michoacana prosperan generalmente en suelos de tipo vertisol y feozem, derivados de materiales geológicos ígneos (principalmente basaltos). Desde el punto de vista climatológico (Anónimo, 1981, 1985), se encuentran en zonas templado subhúmedas del tipo $\mathrm{C}\left(\mathrm{w}_{1}\right)(\mathrm{w})$ y semicálidas subhúmedas $(\mathrm{A}) \mathrm{C}\left(\mathrm{w}_{0}\right)(\mathrm{w})$.

Por otra parte, las áreas donde se ha encontrado esta especie muestran la gran perturbación que han sufrido debido a las actividades antropogénicas, que causan 
cada vez mayor impacto y deterioro al estado natural de estos sitios, lo que probablemente repercute también en la merma de las poblaciones de la planta. Es común observar en sus alrededores diferentes cultivos agrícolas (maíz, sorgo, hortalizas, etc.), tiraderos de basura (en los que hay incendios y otras actividades humanas permanentes), carreteras y/o caminos secundarios, así como pastoreo de ganado equino y bovino principalmente. Tal situación, además de lo escaso y reducido que son las poblaciones de E. michoacana (en las últimas exploraciones llevadas a cabo en varias de las localidades registradas no se han encontrado individuos), han contribuido a la falta de material suficiente para completar su descripción, pues se desconocen las semillas.

Aunque es una planta que se ha registrado únicamente del estado de Michoacán, es muy probable que también exista en Jalisco, ya que la colecta García 7179 pertenece a un sitio relativamente cercano a los límites esta última entidad.

Clave para determinar las especies conocidas de Escobedia en México.

Cáliz con 5 costillas, terminando cada una en el ápice de un diente

E. michoacana

Cáliz con 10 costillas, terminando alternadamente en senos y ápices de los dientes. Hojas lineares; dientes del cáliz linear-lanceolados E. laevis

Hojas foliares lanceoladas a ovadas, a veces angostamente lanceoladas a más o menos lineares; dientes del cáliz triangular-deltoideos a ovados.

Hojas angostamente lanceoladas a más o menos lineares; brácteas situadas en la porción media del pedúnculo E. guatemalensis Hojas más anchas, lanceoladas, ovadas o elíptico-lanceoladas; brácteas situadas hacia la porción superior del pedúnculo.

Tubo del cáliz de 2.5 a 3(4) cm de largo; pedúnculo de 6 a $10 \mathrm{~cm}$ de largo; hojas ovadas a lanceoladas, de 2.5 a $4 \mathrm{~cm}$ de largo

E. peduncularis

Tubo del cáliz de 4 a $6 \mathrm{~cm}$ de largo; pedúnculo de 1 a $6 \mathrm{~cm}$ de largo; hojas elíptico-lanceoladas u ovadas, de 5 a $11 \mathrm{~cm}$ de largo.

Pedúnculo evidentemente claviforme, de 1 a $3 \mathrm{~cm}$ de largo; tubo de la corola de hasta $8 \mathrm{~cm}$ de largo, glabro u ocasionalmente con algunos pequeños pelos glandulares; brácteas situadas inmediatamenteabajo del cáliz E. crassipes 
Pedúnculo no o inconspicuamente claviforme, de 2 a $6 \mathrm{~cm}$ de largo; tubo de la corola de 10 a $13 \mathrm{~cm}$ de largo, externamente glandulosopubescente, sobre todo en las nervaduras principales, llegando en ocasiones hasta el limbo; brácteas situadas 2 a $5 \mathrm{~mm}$ abajo del cáliz E. longiflora

\section{AGRADECIMIENTOS}

Se agradece a los curadores de los herbarios CIMI, EBUM, ENCB, IBUG, IEB, MEXU y XAL por permitir consultar sus acervos. Igualmente a Ignacio García del CIIDIR-Jiquilpan (CIMI), por compartir información de algunas colectas. A los Drs. J. Rzedowski y V. W. Steinmann por la revisión crítica del manuscrito, así como al Sr. Rogelio Cárdenas por la elaboración de la ilustración y finalmente, al Instituto de Ecología, A.C. (cuenta 20006), por el apoyo otorgado para llevar a cabo este trabajo.

\section{LITERATURA CITADA}

Anónimo. 1981. Atlas nacional del medio físico. Secretaría de Programación y Presupuesto. México, D.F. 224 pp.

Anónimo. 1985. Síntesis geográfica de Michoacán. Secretaría de Programación y Presupuesto, Instituto Nacional de Estadística, Geografía e Informática. México, D.F. 316 pp.

Holmgren, N. H. y U. Molau. 1984. Scrophulariaceae. In: Harling, G. y B. Sparre (eds.). Flora of Ecuador. University of Göteborg - Riksmuseum. Estocolmo. Vol. 21. pp. $148-151$.

Olmstead, R. G., C. W. de Pamphilis, A. D. Wolfe, N. D. Young, W. J. Elisons \& P. A. Reeves. 2001. Desintegration of the Scrophulariaceae. Amer. Journ. Bot. 88(2): 348-361.

Pennell, F. 1931. Escobedia. A neotropical genus of Scrophulariaceae. Proc. Acad. Nat. Sci. Philadelphia 83: 411-426.

Ulloa, C. 2006. Aromas y sabores andinos. In: Moraes, M., B. Ollgard, L. P. Kvist, F. Borchsenius \& H. Balslev (eds.). Botánica económica de los Andes Centrales. Universidad Mayor de San Andrés. La Paz. p. 319. 
